\title{
Nonlinear Wave Packets of Equatorial Kelvin Waves
}

\author{
Guan-yu Chen \\ Institute of Harbour and Marine Technology \\ Wuchi, Taichung 435 \\ Taiwan
}
John P. Boyd
Department of Atmospheric, Oceanic
and Space Science and Laboratory for Scientific Computation, University of Michigan, 2455 Hayward Avenue, Ann Arbor MI 48109
jpboyd@engin.umich.edu;
http://www.engin.umich.edu:/ jpboyd/

September 27, 2000

\begin{abstract}
The weakly nonlinear dynamics of packets of equatorial Kelvin waves is studied using singular perturbation theory applied to the shallow water wave equations. Within the limits of the perturbation theory, which is formally restricted to weak mean shear and weak nonlinearity, we derive a Nonlinear Schroedinger equation to describe the envelope of the wave packet. We find that nonlinearity has a defocusing effect so that coherent wave packets must owe their existence entirely to the generation mechanism rather than to nonlinear focusing of a broad initial disturbance.
\end{abstract}

\section{Introduction}

\subsection{Oceanographic Relevance of Nonlinear Wavepackets}

The Kelvin wave is the gravest and most energetic latitudinal mode in the tropical ocean. It would seem self-evident that the nonlinear dynamics of the Kelvin wave needs to be understood as thoroughly as possible. In this article, we analyze the dynamics of a nonlinear Kelvin wave packet whose structure in longitude is illustrated schematically in Fig 1.

The strongest motivation for our study would be direct observations of such wavepackets. Satellite altimeter data such as Fig. 2 and similar figures in Zheng, 


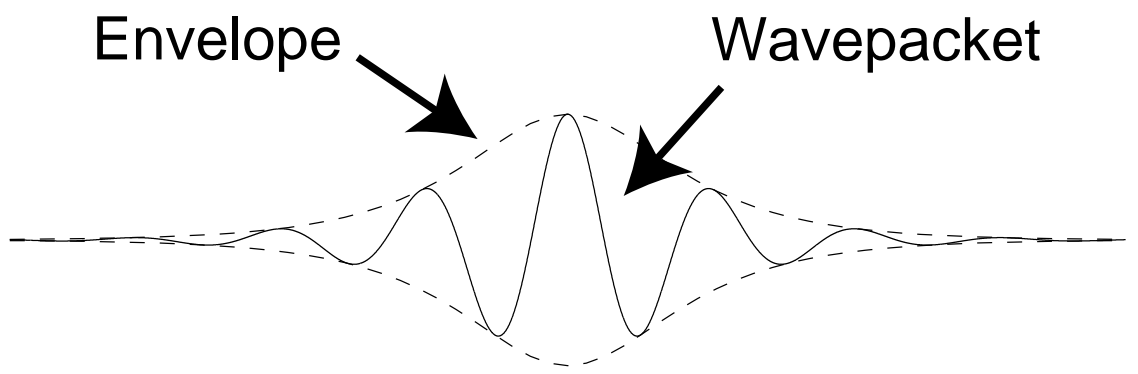

Figure 1: A wavepacket (solid) is a disturbance which is the product of a "carrier wave", $\cos (k[x-c(k) t])$, with an "envelope" function (dashed). The Nonlinear Schrödinger equation most accurately models weakly nonlinear wavepackets when the envelope varies slowly compared to the sinusoidal oscillations of the carrier wave.

Yan, Ho and Tai(1995) certainly suggest such wavepackets. Because the Kelvin wave is the lowest latitudinal mode, it tends to be excited more strongly than high modes by arbitary forcing, so presumbably Kelvin waves contribute a large part of the signal and perhaps dominate it. However, identifying individual packets is hard. Indeed, similar packets (of baroclinically unstable waves) have been identified in the atmosphere only within the last five years even though meteorologists have always been blessed with much richer data sets than physical oceanographers (Lee and Held, 1993, Esler, 1997). Furthermore, a wave packet varies on both a fast length scale (that of the "carrier" wave) and a slow length scale (that of the "envelope"). Resolving two length scales (and the corresponding time scales) is beyond the capacity of present-day observing systems.

A more elemental question is: Does the Nonlinear Schrödinger (NLS) equation derived here apply to any real-world waves. Here, the answer is an unqualified Yes. In fiber optics telecommunication, the prediction of envelope solitary waves by Hasegawa and Tappert (1973) was triumphantly confirmed in laboratory experiments by Mollenauer, Stolen and Gordon (1980). The significance of this for future telephone and Internet systems is that, for packets of infinitesimal amplitude, communication rates are limited by dispersion, which eventually causes the packets to spread so wide that Mrs. Jones' conversation overlaps Mr. Smith's. In envelope solitary waves, the dispersion is counteracted by nonlinear self-focusing so that the packets can in principle remain coherent forever. Soliton patterns gave no degradation even after repeatedly circling a loop of fiber for a total travel distance of 20,000 kilometers (Desurvire, 1994)! Glass (1993) predicts: "Solitons, with all of their advantages, are expected to find their way into commercial systems before the end of the decade."

In fluid mechanics, envelope solitary waves have been observed in ship-wave wakes (Brown et al., 1989). Yuen and Lake (1975) and Lake, Yuen, Rungadier and Ferguson(1977) and Hammack (reviewed in Ablowitz and Segur, 1981) have 


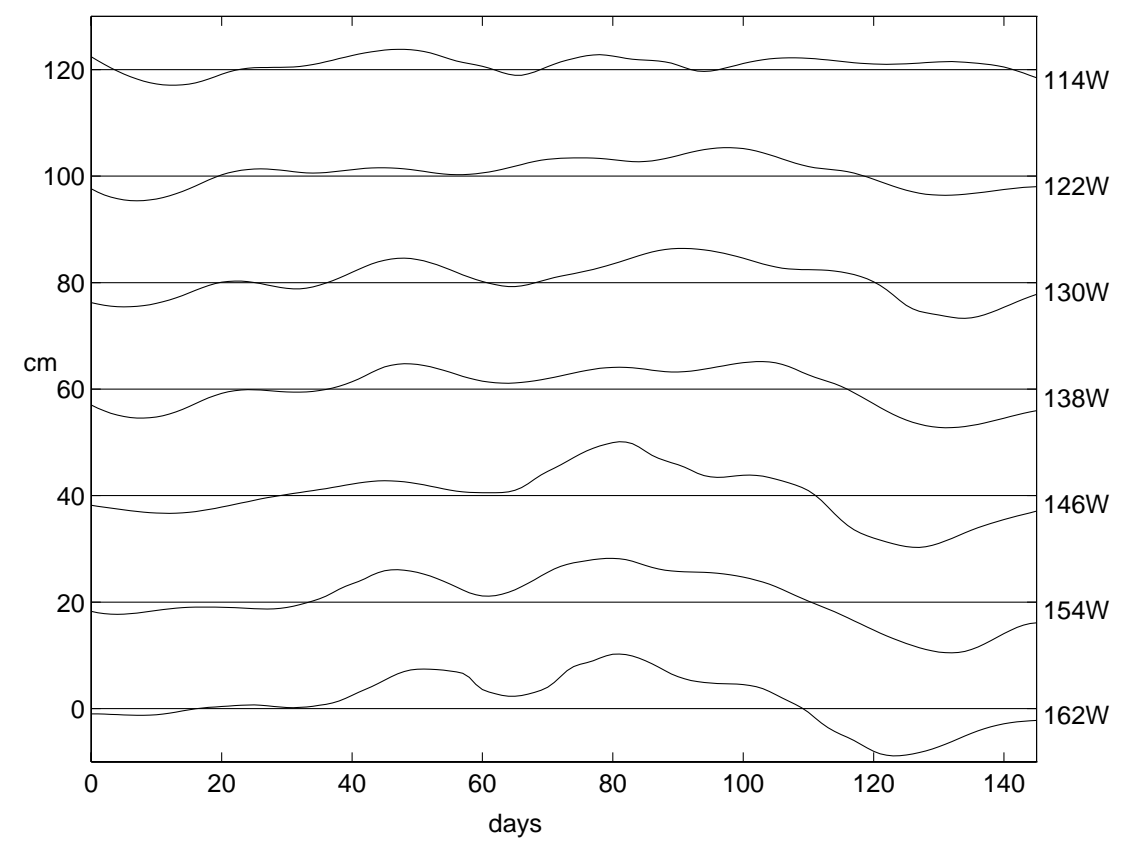

Figure 2: Time series of Geosat SLA data at 7 locations along the equator, October, 1985 through February 1986. The horizontal lines mark the zeros at each longitude; each dividing line is separated by $20 \mathrm{~cm}$ of sea level, which thus provides a scale for the curves. From Miller, Cheney and Douglas (1988) with permission of the authors and Science magazine. 
shown that there is good agreement between the NLS theory and water tank experiments for nonlinear packets of deep water waves, both for envelope solitary waves and for the sideband or "Benjamin-Feir" instability.

Boyd (1983c) has shown that the Nonlinear Schrödinger equation also applies to equatorial Rossby, Yanai and gravity waves under the same restrictions as for other applications of this model: Weak nonlinearity and a good separationof-scales between the envelope and carrier wave. Furthermore, the theory of linear dispersive waves shows that an arbitrary initial disturbance will eventually disperse into a continously-varying wave packet of the kind describable, for weak nonlinearity, by the NLS equation (Whitham, 1974). It follows that unless special conditions apply, the equatorial Kelvin wave will form wavepackets of NLS type.

There are two such special conditions for oceanic Kelvin waves. The first is that in the absence of a mean current, an equatorial Kelvin wave is nondispersive, and its nonlinear behavior is frontogenesis and wave breaking (Boyd, 1980, Ripa, 1982, 1985). Boyd(1998b) and Fedorov and Melville(2000) showed that the front is complicated by a resonance with eastward-travelling gravity waves, which causes the front to curve westward with increasing distance from the equator. (In the language of (Boyd, 1998a, 1999), the front is "weakly nonlocal" because of the trailing oscillations in the lowest symmetric gravity wave.) However, Boyd(1984) and Long and Chang(1990) have shown by explicit calculation that mean shear currents induce dispersion in the Kelvin wave. We shall try to answer the question: Is this shear-induced dispersion strong enough to be oceangraphically significant?

The other special condition is that the oceans are bounded by coasts. Given enough time, weak nonlinearity is always significant in reshaping wave packets. Because of coastal boundaries, however, Kelvin wave packets may not have enough time for either dispersion or nonlinearity to be important before coastal reflection shreds the equatorial Kelvin wave into a mix of coastal Kelvin waves and reflected Rossby modes.

It is unsatisfying that the observations are inadequate to precisely characterize Kelvin wave packets. However, Delcroix, Picaut and Eldin (1991), Johnson and McPhaden (1993) and Kessler, McPhaden and Weickmann(1995) have documented the importance of oceanic equatorial Kelvin waves. Fedorov and Melville (2000), reviewing these observations, conclude the ratio of wave amplitude to thermocline depth is the order of $10 \%$ to $30 \%$. We shall see below that much weaker amplitude is sufficient for nonlinearity to make major changes in wave packets. The neglect of nonlinearity and dispersion for equatorial Kelvin waves is wishful thinking. 


\subsection{Shallow Water Model}

The starting point of our study is the nonlinear shallow water wave system on the equatorial beta-plane:

$$
\begin{gathered}
u_{t}+u u_{x}+v u_{y}-y v+\phi_{x}=0 \\
v_{t}+u v_{x}+v v_{y}+y u+\phi_{y}=0 \\
\phi_{t}+u_{x}+v_{y}\left\{(u \phi\}_{x}+\{v \phi\}_{y}=0\right.
\end{gathered}
$$

where $u$ and $v$ are east-west and north-south velocities and $\phi$ is the deviation from mean depth where the total depth of a fluid column is $h=1+\phi$. As in Moore and Philander (1977), these equations have been nondimensionalized in terms of "Lamb's parameter"

$$
\tilde{E}=\frac{4 \Omega^{2} a^{2}}{g H}
$$

where $\Omega$ is the angular frequency of the earth's rotation, $a$ is the radius of the earth, and $H$ is the equivalent depth. The nondimensional length scale $L$ and time scale $T$ are

$$
L=a \tilde{E}^{-1 / 4}, \quad T=\tilde{E}^{1 / 4}(2 \Omega)^{-1}
$$

This model can be interpreted as a "one-and-a-half-layer" model; it correctly describes the dynamics of two layers of fluid if the bottom layer is infinitely deep and at rest and the equivalent depth $H$ is set equal to the mean depth of the upper layer multiplied by the fractional density difference between the two layers.

Because the tropical ocean has a sharp thermocline dividing a well-mixed upper layer of $O(100)$ meters depth from the colder fluid below, roughly forty times thicker, the shallow water model has been widely used in tropical oceanography.

Marshall and Boyd (1987) have derived the NLS equation for equatorial waves in a continuously stratified fluid. The additional vertical degrees of freedom alters little. However, nonlinear effects are slightly weakened because the self-interaction of the wave projects partly onto higher baroclinic modes, which are not resonant with the first baroclinic mode.

\subsection{Overview of Theory}

The derivation of the Nonlinear Schrödinger (NLS) model has three parts. First, the standard NLS-yielding perturbation scheme is disrupted by the (almost) nondispersive character of the Kelvin wave, which creates a resonance between the carrier wave in the Kelvin mode and the long wave and second harmonics in the Kelvin mode. This Kelvin-Kelvin resonance actually simplifies the

derivation because it allows one to neglect all latitudinal modes in the nonlinear 
interaction except the Kelvin mode itself. This in turn implies that the nonlinear effects can be deduced directly from the Korteweg-deVries equation (KdV) model of the Kelvin wave (Boyd, 1984).

Second, the linear dispersion of the Kelvin wave (as opposed to dispersion caused by nonlinear self-interaction) is generated entirely by the latitudinally varying zonal mean flow. In Boyd (1984), this shear-induced dispersion was calculated in the limits of long zonal wavelength. It controls the coefficient of the third derivative in the Korteweg-deVries model for the weakly nonlinear Kelvin wave in shear. For arbitrary zonal wavenumber $k$, the effects of mean shear (or anything else) may produce a complicated phase speed $c(k)$. However, as will be shown in Section 3, that to second order in the strength of the shear $\delta, c(k)$ is a quadratic polynomial in the wavenumber, identical with the long wave approximation to the dispersion relation.

Collectively, these simplifications imply that it is not necessary to derive the NLS equation from the equatorial shallow-water equations. (Such a derivation is given in full for the sake of completeness in Chen(1998).) Instead, the NLS equation can be derived from the much simpler Korteweg-deVries model of Boyd(1984). The third stage is to execute this KdV-to-NLS step.

The perturbative analysis depends on three parameters: (i) the amplitude $\epsilon$ (ii) the inverse of the length scale $L$ of the envelope and (iii) the strength of the latitudinal shear $\delta$. It is physically legitimate to choose these parameters independently of one another so that the perturbative approximation is a triple series.

However, if $\epsilon<<1 / L$, then the envelope will simply disperse and nonlinearity will never be significant; if $\epsilon>>1 / L$, then dispersion can be neglected and the wave will steepen and break. It follows that the interesting regime is when $\epsilon \sim O(1 / L)$ so that dispersion and nonlinearity are of comparable importance. In what follows, we shall restrict ourselves to this regime. This collapses the number of independent perturbation parameters to two: amplitude/dispersion parameter $\epsilon$ and shear strength $\delta$.

\section{Simplification of Nonlinearity Through Near- Resonance}

At lowest order in both the Korteweg-deVries and Nonlinear Schrödinger theories, the Kelvin wave is of the form

$$
u=\phi=\frac{1}{2} A(x, t) \exp \left(-\frac{1}{2} y^{2}\right)
$$

The nonlinear terms in the shallow water wave equation are all quadratic and thus proportional to $\exp \left(-y^{2}\right)$. The expansion of $\exp \left(-y^{2}\right)$ as a series of equatorial modes is an infinite series. Thus, the first order perturbative corrections are a sum over all modes. The same is true for wavepackets of other species of equatorial waves (Boyd, 1983a). 
There is one crucial difference between Kelvin wavepackets and other species of equatorial waves. In the absence of mean shear, the Kelvin wave is nondispersive. This implies that the self-interaction of the Kelvin wave travels (again neglecting shear) at exactly the same speed as the Kelvin wave itself. This creates a self-resonance of the Kelvin wave. In the absence of mean currents, the Kelvin wave can never propagate with a steady shape, but instead must deform continuously as it propagates. The result is frontogenesis and wave-breaking (Boyd, 1980a, Ripa, 1982).

With mean currents, the Kelvin wave is dispersive, but only weakly so. If the strength of the mean currents is a small parameter $\delta$, then the first order Kelvin corrections will be larger than those of all the other equatorial modes by $O\left(1 / \delta^{2}\right)$. Because of the near-resonance,the first order response is dominated by the interaction of the Kelvin mode with itself. It is thus legitimate, to within a relative error of $O\left(\delta^{2}\right)$, to neglect all the other modes at first order and retain only the projection of the Kelvin nonlinearity onto the Kelvin mode.

The mean shear distorts the structure of the Kelvin mode in the sense that if the shallow water wave equations are linearized about a mean flow of magnitude $O(\delta)$, the eigenmode is no longer simply a Gaussian in latitude as in Eq. 6 . However, the distortions in shape are $O(\delta)$. It follows that it is consistent with a perturbative treatment of shear to ignore these shear-induced structural changes to lowest order in calculating the Kelvin self-interaction and its projection onto the Kelvin mode.

This implies a remarkable simplification in the treatment of nonlinearity: all equatorial modes can be neglected except the Kelvin mode itself. The KortewegdeVries model of Boyd(1984), which includes only the Kelvin-Kelvin interaction, thus successfully captures all the nonlinear interaction that appears in the Nonlinear Schrödinger model of Kelvin wavepackets.

This claim can be justified in a couple of other ways. The first is to derive the Nonlinear Schrödinger equation directly from the shallow water wave equations, and then compare the result with the Schrödinger coefficients as derived from the KdV equation. Chen (1998) has shown that these two approaches do indeed give identical results.

The second way is to recall that the normal modes of the equatorial shallow water equations, linearized about a state of rest, are a complete orthogonal set. Anderson(1973), Tribbia(1984), Boyd(1998b) and many authors in tropical oceanography have built numerical models using a latitudinal basis of these equatorial normal modes. As the truncation of this infinite set to a finite number of functions $N$ increases, the error in this "Hough-Hermite" expansion may be made arbitrarily small.

The most drastic truncation is to take $N=1$, that is, a single latitudinal basis function. The complete solution may be then be represented in the form of Eq. 6. Projecting the shallow water wave equations onto this one mode ("Galerkin method") gives an equation for the amplitude:

$$
A_{t}+A_{x}+\sqrt{3 / 8} A A_{x}=0 \quad \text { [One-Mode, Kelvin-Only Galerkin Model] }
$$


The good news is that this one-mode model correctly reproduces the nonlinearity of the Kelvin mode to lowest order in both the KdV and NLS theories. When $A(x, t)$ is a wavepacket, the equation for the envelope of the wave packet is the Nonlinear Schrödinger equation, and the nonlinear coefficient of this envelope equation is correctly derivable from the Kelvin-only Galerkin model, Eq. 7.

The bad news is that the $N=1$ Galerkin model is still nondispersive: if $A(x, t)$ includes a non-propagating, $x$-independent component $A_{0}$, then the effect is merely to shift the phase speed from unity to $c_{0}=1+A_{0}$, independent of zonal wavenumber. What has gone wrong?

The answer is that our argument for neglecting non-Kelvin modes is applicable only to propagating components. The non-Kelvin contributions to the nonlinearity will be smaller by $O\left(\epsilon \delta^{2}\right)$ than the dominant Kelvin-Kelvin nonlinearity. However, for the shear-induced perturbation, both the Kelvin and non-Kelvin terms are $O\left(\delta^{2}\right)$ relative to the unperturbed fundamental. It follows that for the mean flow, we must consider all latitudinal modes as done in the next section.

\section{Shear-Induced Dispersion Through Perturba- tion Theory}

The crucial simplification is that the calculation of the shear effects is completely independent of that for nonlinearity. Indeed, the formalism of the computation of shear effects is identical to that for linearized waves that are sinusoidal in $x$ (rather than wave packets), as in Boyd(1978a,b).

The small parameter $\delta$ for the shear flow is the multiplier of the mean current, which is

$$
u_{0}=\delta U(y)
$$

The mean flow is assumed to be in geostrophic balance, implying

$$
\Phi=-\int y^{\prime} U\left(y^{\prime}\right) d y^{\prime}
$$

It is convenient to shift into the "sum" variable $S$ and "difference" variable $D$ in place of $u$ and $\phi$ where

$$
S \equiv \phi+u, \quad D \equiv \phi-u
$$

We then expand all the unknowns as

$$
\begin{aligned}
S & =\left\{S^{(0)}+\delta S^{(1)}+S^{(2)}+\ldots\right\} \exp (i[k x-\omega(k) t]) \\
v & =\left\{v^{(0)}+\delta v^{(1)}+v^{(2)}+\ldots\right\} \exp (i[k x-\omega(k) t]) \\
D & =\left\{D^{(0)}+\delta D^{(1)}+D^{(2)}+\ldots\right\} \exp (i[k x-\omega(k) t])
\end{aligned}
$$


The advantage of the new variables is that for the unperturbed Kelvin wave, only the sum variable is nonzero:

$$
S^{(0)}=\exp \left(-(1 / 2) y^{2}\right), \quad v^{(0)} \equiv 0, \quad D^{(0)} \equiv 0
$$

The frequency is similarly expanded as

$$
\omega \equiv k c=k\left\{c^{(0)}+\delta c^{(1)}+\delta^{2} c^{(2)}+\ldots\right\}
$$

where $c$ is the phase speed.

The $O(\delta)$ perturbation equations are

$$
\begin{aligned}
v_{y}^{(1)}-y v^{(1)} & =-i k\left\{U(y)+\frac{1}{2} \Phi(y)-c^{(1)}\right\} S^{(0)} \equiv f_{1} \\
S_{y}^{(1)}+y S^{(1)}-2 i v^{(1)}+D_{y}^{(1)}-y D^{(1)} & =0 \\
-v_{y}^{(1)}-y v^{(1)}+2 i k D^{(1)} & =i k \frac{1}{2} \Phi(y) S^{(0)}
\end{aligned}
$$

By direct substitution, the zeroth order Kelvin wave solves the homogeneous version of this set. This implies that the inhomogeneous equation has a bounded solution only if the inhomogeneous terms satisfy a solvability condition, which is that the inhomogeneous terms must be orthogonal to the Kelvin mode:

$$
\int_{-\infty}^{\infty} d y \exp \left(-(1 / 2) y^{2}\right) f_{1}=0
$$

where $f_{1}$ is the right-hand side of the first equation in the $O(\delta)$ set. This determines the correction to the phase speed as

$$
c^{(1)}=\frac{1}{\sqrt{\pi}} \int_{-\infty}^{\infty} \exp \left(-y^{2}\right)\left\{U(y)+\frac{1}{2} \Phi(y)\right\} d y
$$

Note that $c^{(1)}$ is independent of $k$, and therefore nondispersive. To obtain dispersion, it is necessary to continue the perturbation series to the solvability condition at $O\left(\delta^{2}\right)$.

The first equation in (16) is a first order, inhomogeneous equation for $v^{1}$ alone. It can be solved by quadratures. One can then solve the remaining equations for $D^{1}$ and $S^{1}$ in turn, also by quadratures:

$$
\begin{aligned}
S^{1} & =\left\{f_{S}(y)+\tilde{f}_{S}(y) k^{2}\right\} S^{0} \\
v^{1} & =i k f_{v}(y) S^{0} \\
D^{1} & =f_{D}(y) S^{0}
\end{aligned}
$$

where

$$
f_{v}=\exp \left(y^{2}\right) \int_{-\infty}^{y} \exp \left(-z^{2}\right)\left\{c_{1}-U(z)-\frac{1}{2} \Phi(z)\right\} d z
$$




$$
\begin{gathered}
f_{S}=\int_{-\infty}^{y}\left\{\frac{1}{2} z \Phi(z)+z f_{v, z}(z)-\frac{1}{4} \Phi_{z}(z)-\frac{1}{2} f_{v, z z}(z)\right\} d z \\
\tilde{f}_{S}=-2 \int_{-\infty}^{y} f_{v}(z) d z \\
f_{D}(y)=\frac{1}{2} f_{v, y}(y)+\frac{1}{4} \Phi(y)
\end{gathered}
$$

The solvability condition at second order gives

$$
c^{(2)}=c_{\text {nondispersive }}^{(2)}+\Omega k^{2}
$$

where

$$
\begin{gathered}
c_{\text {nondispersive }}^{(2)}=\frac{1}{\sqrt{\pi}} \int_{-\infty}^{\infty} \exp \left(-y^{2}\right)\left\{f_{v}(y) U_{y}+f_{S}(y)\left(\frac{1}{2} \Phi(y)-c_{1}\right)\right. \\
+\frac{3}{4} f_{v, y}(y) \Phi(y)+f_{S}(y) U(y)+f_{v}(y) \Phi_{y} \\
\left.-y f_{v}(y) \Phi(y)-\frac{1}{8} \Phi^{2}(y)\right\} d y \\
\Omega=\frac{1}{\sqrt{\pi}} \int_{-\infty}^{\infty} \exp \left(-y^{2}\right)\left\{U(y)+\frac{1}{2} \Phi(y)-c_{1}\right\} \tilde{f}_{S}(y) d y
\end{gathered}
$$

It is unnecessary to compute the second order corrections to $S$, $v$, and $D$.

\section{Derivation of NLS from $\mathrm{KdV}$}

The KdV equation for the nonlinear Kelvin wave is, neglecting small shearinduced $O(\delta)$ corrections to the nonlinear coefficient,

$$
A_{t}+c_{\text {nondispersive }} A_{x}+\sqrt{3 / 8} A A_{x}-\Omega \delta^{2} A_{x x x}=0
$$

where

$$
c_{\text {nondispersive }}=1+c^{(1)} \delta+c_{\text {nondispersive }}^{(2)} \delta^{2}
$$

with coefficients that depend on the mean shear flow assumed with $\delta$ as the strength of the shear flow. It is convenient to shift into a frame of reference moving with the wave by defining

$$
X \equiv x-c_{\text {nondispersive }}{ }^{t}
$$


The KdV equation is then simplified to

$$
A_{t}+\sqrt{3 / 8} A A_{X}-\Omega \delta^{2} A_{X X X}=0
$$

The KdV equation has a wide range of solutions including solitary waves whose shape is proportional to the square of the hyperbolic secant function (Boyd, 1984). To focus on weakly nonlinear wavepackets, we shall assume that the lowest order solution is of the form

$$
A(x, t) \sim \epsilon A_{01}(\zeta, \tau) \exp (i[k X-\omega(k) t])
$$

where $\epsilon<<1$ is a small parameter, $k$ is the zonal wavenumber of the "carrier wave" and its frequency $\omega(k)$ is specified by the linear dispersion relation for Kelvin waves in a mean shear flow and where

$$
\zeta \equiv \epsilon\left(X-c_{g}(k) t\right)
$$

where $c_{g}(k)$ is the group velocity, given by the linear dispersion relation as $\partial \omega / \partial k$ and where

$$
\tau=\epsilon^{2} t
$$

In words, the wavepacket at lowest order is the product of two factors (Fig. 1). The sinusoidal factor $\exp (i[k x-\omega(k) t])$ is called the "carrier wave" or "fundamental"; its peaks and troughs propagate at the phase velocity $c_{p}=\omega / k$. The factor $A_{01}(\zeta, \tau)$ is the "envelope" and it propagates at roughly the linear group velocity. The adjective "roughly" is appropriate because the dependence on the slow time variable $\tau$ allows for small nonlinear alterations in the speed of the envelope. It is the envelope, and not the complete longitude-and-time-factor $A(X, t)$, which will turn out to solve the Nonlinear Schrödinger equation.

There is nothing particular unusual or mysterious about the assumed form of the wavepacket. AM (Amplitude Modulation) radio is transmitted in the form of such packets. A radio station transmitting at 550 on AM dial is generating a carrier wave with a frequency $\omega=550,000$ cycle per second (that is, 550 kilohertz). The sounds of music and human voices are transmitted by modulating the envelope at much lower frequencies. To transmit a pure tone that is "middle C" (512 hertz), the envelope $A_{01}(\zeta, \tau)$ is oscillated sinusoidally at a frequency a little smaller than $1 / 1000$ that of the carrier wave.

The scale separation between the slow variations of the envelope and much faster oscillations of the carrier wave is embodied in the factor of the perturbation parameter $\epsilon$ in the definition of the envelope spatial variable $\zeta$, which is therefore a "slow" variable whenever $\epsilon<<1$. Such wave packets are well-defined even when the amplitude is infinitesimal and can still be analyzed through the perturbation technique known as the "method of multiple scales" in this limit. The Nonlinear Schrödinger equation is obtained in the slightly more complicated case that the amplitude of the packet is not infinitesimal so that weak nonlinear effects occur. 
The expansion for a wavepacket is of the form

$$
A=\epsilon \sum_{j=0}^{\infty} \epsilon^{j} \sum_{m=0}^{j+1} A_{j m}(\zeta, \tau) E^{m}+\text { c. c. }
$$

where $E$ is the carrier wave,

$$
E(X, t)=\exp (i[k X-\omega(k) t])
$$

where "c. c." denotes the complex conjugate of the terms in the sum.

Derivatives are interpreted by the chain rule:

$$
\begin{gathered}
\frac{\partial}{\partial t} \rightarrow \frac{\partial}{\partial t}-\epsilon c_{g} \frac{\partial}{\partial \zeta}+\epsilon^{2} \frac{\partial}{\partial \tau} \\
\frac{\partial}{\partial X} \rightarrow \frac{\partial}{\partial X}+\epsilon \frac{\partial}{\partial \zeta}
\end{gathered}
$$

In a minor abuse of notation, we retain the same symbol for both the original coordinates $X, t$ and for the corresponding "fast" coordinates; the fussy reader may introduce a fast variable $t^{\prime} \equiv t$ and then add primes to $t$ on the right side of (37).

Substituting Eqs. $(35,37,38)$ into Eq. (31) gives a sequence of equations at each order.

$$
O(\epsilon E): \quad-i A_{01}\left(-\Omega \delta^{2} k^{3}+\omega\right)=0
$$

which implies the linear dispersion relation (in the moving reference frame)

$$
\omega(k)=\Omega \delta^{2} k^{3}
$$

At $O\left(\epsilon^{2}\right)$, the quadratic nonlinearity of $A_{01} \exp (i k X-i \omega t)+$ complex conjugate gives a "second harmonic" $A_{12} \exp (2 i k X-2 i \omega t)+$ complex conjugate, in which the wavenumber and frequency of the carrier wave are both doubled compared to that of the fundamental, plus a "long wave" $A_{10}(\zeta, \tau)$, which is independent of the fast length and space scales.

$$
O\left(\epsilon^{2} E^{2}\right): \quad 6 i \Omega \delta^{2} k^{3} A_{12}+i \sqrt{3 / 8} k\left(A_{01}\right)^{2}=0
$$

which implies

$$
A_{12}=-\frac{\sqrt{3 / 8}}{6 \Omega \delta^{2} k^{2}}\left(A_{01}\right)^{2}
$$

Because the long wave component is independent of the fast scales, it is determined by the $O\left(\epsilon^{3}\right)$ equation even though the long wave itself is $O\left(\epsilon^{2}\right)$.

$$
O\left(\epsilon^{2} E^{0}\right): \quad-3 \Omega \delta^{2} k^{2}\left(A_{10}\right)_{\zeta}+\sqrt{\frac{3}{8}}\left(\left|A_{01}\right|^{2}\right)_{\zeta}=0
$$


(where the asterisk denotes complex conjugation) whose solution is

$$
A_{10}=\frac{\sqrt{3 / 8}}{3 \Omega \delta^{2} k^{2}}\left|A_{01}\right|^{2}
$$

The interaction of the $O(\epsilon)$ fundamental with the $O\left(\epsilon^{2}\right)$ long wave and second harmonics excites terms proportional to $E$. The solvability condition is that these terms sum to zero, giving the Nonlinear Schrödinger (NLS) equation:

$$
i\left(A_{01}\right)_{\tau}+3 \Omega \delta^{2} k\left(A_{01}\right)_{\zeta \zeta}-\frac{1}{16 \Omega \delta^{2} k}\left|A_{01}\right|^{2} A_{01}=0
$$

The equation can be written in the alternative form

$$
i\left(A_{01}\right)_{\tau}+\frac{1}{2} \frac{d^{2} \omega}{d k^{2}}\left(A_{01}\right)_{\zeta \zeta}-\frac{1}{16 \Omega \delta^{2} k}\left|A_{01}\right|^{2} A_{01}=0
$$

where the nonlinear coefficient or "Landau constant" $\nu$ is

$$
\nu=-\frac{1}{16 \Omega \delta^{2} k}
$$

and the second derivative of the dispersion relation gives

$$
\frac{1}{2} \frac{d^{2} \omega}{d k^{2}}=3 \Omega \delta^{2} k \quad=-\frac{3}{16} \frac{1}{\nu}
$$




\title{
LONG WAVE
}

\author{
$\left|A_{01}\right|^{2}$
}
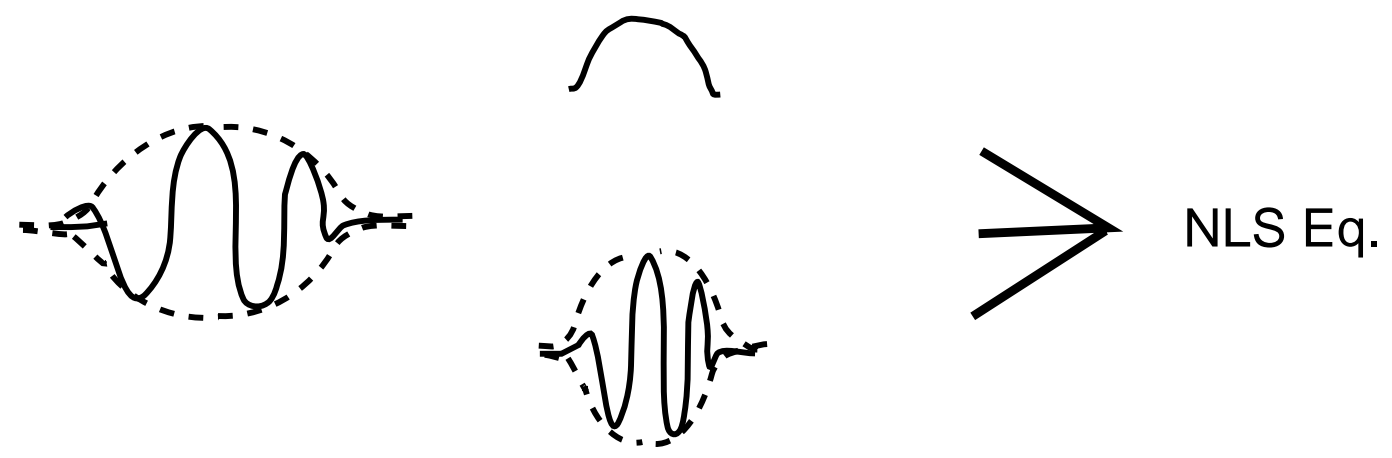

A01 exp(ikx-iwt) $\quad A_{01}{ }^{2} \exp (2 i k x-2 i w t)$

\section{FUNDAMENTAL SECOND HARMONIC}

$\mathrm{O}(\varepsilon)$

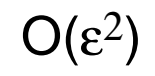

$\mathrm{O}\left(\varepsilon^{3}\right)$

Figure 3: Schematic of the perturbation theory in powers of the small parameter $\epsilon$. The fundamental is the product of the slowly-varying envelope $A_{01}\left(\epsilon\left[x-c_{g} t\right], \epsilon^{2} t\right)$ with the carrier wave $\exp (i k x-i w t)$. The self-interaction of the fundamental excites two components whose amplitude is $O\left(\epsilon^{2}\right)$ : a long wave component, which varies only slowly with longitude and time, and a second harmonic, whose envelope and carrier wave both have length and time scales half that of the fundamental. At $O\left(\epsilon^{3}\right)$, the interaction of the $O\left(\epsilon^{2}\right)$ components with the fundamental forces the envelope $A_{01}$ to evolve as a solution to the Nonlinear Schrödinger equation. 


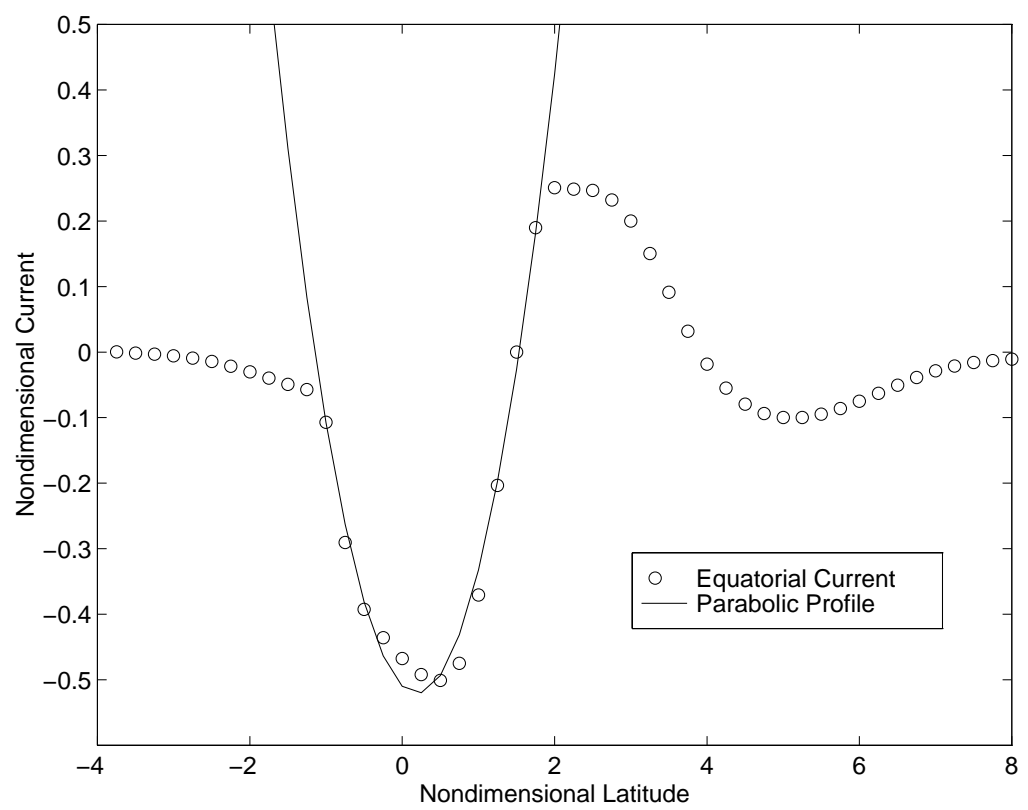

Figure 4: Comparison of the Unsymmetrical-Parabola (UP) mean flow (solid) with observed currents (circles).

\section{Mean Current: Observations and Uncertainty}

Fig. 4 shows the fit of observed currents by the analytic function

$$
U(y)=0.290 y^{2}-0.112 y-0.510 \quad \text { ["Unsymmetrical-Parabola" (UP)] }
$$

which we shall henceforth dub the "Unsymmetrical-Parabola" jet. Although this is unbounded, the integrals in the perturbation theory are all finite; the Gaussian factor in the Kelvin wave ensures that the mode effectively sees only the behavior of the jet close to the equator.

The finite difference numerical model, however, requires a bounded mean flow. We employed

$U=-(1 / 2) \exp \left(-[25 / 64] y^{2}\right)\left(1-[25 / 64] y^{2}\right)\left(1+[25 / 36] y^{2}\right)$

["Gaussian-Quartic" (GQ)]

which is very similar to the Unsymmetrical-Parabola near the equator.

To translate dimensional flow into the nondimensional variables defined by Eq. (5), we set

$$
L=300 \mathrm{~km}, \quad T=1.7 \text { days }
$$

Ocean currents are depth-dependent, of course, but this profile is reasonable for the one-and-a-half layer model used here. Because the Kelvin wave is equato- 
rially trapped, the shape of $U(y)$ at high latitudes is irrelevant, and so our fit has been chosen to be an accurate approximation only close to the equator.

One obvious question is: How sensitive are the coefficients of the NLS equation to the mean current? In the perturbation theory, the mean current was written as, denoting the circumference of the earth by $C_{\text {earth }}$

$$
\frac{1}{C_{\text {earth }}} \int u(x, y) d x \equiv u_{0} \equiv \delta U(y)
$$

Thus, the strength of the mean flow can be varied, without altering the shape of the current, by varying the shear strength parameter $\delta$. Eqs. 47 and 48 then show that the ratio of the nonlinear coefficient to the dispersive coefficient of the NLS equation is

$$
\mathcal{N} \equiv \frac{\nu}{(1 / 2) \omega_{k k}}=-\frac{1}{48 \Omega^{2} \delta^{4} k}
$$

This implies that the ratio of coefficients depends on the fourth power of the strength of the shear. Thus, even a small change in the strength of the shear will drastically alter the coefficients of the NLS equation.

In the perturbation theory described earlier, the dispersion parameter $\Omega$ is independent of the carrier wavenumber $k$ to second order in the strength of the shear. Unfortunately, this independence is not true in general. We calculated $\Omega$ a second, non-perturbative way by numerically solving the eigenvalue problem. Fig. 5 shows that for a smooth current similar to the observed mean flow, $\Omega$ varies rapidly with $k$. The Kelvin wave in such a shear is almost nondispersive at $k=2$ where $\Omega(k)$ is very small. The large variations in $\Omega$ imply the shear perturbation theory, which predicts that $\Omega(k)$ should be simply a horizontal line, is clearly not very accurate.

\section{Generic Behavior of NLS Wave Packets}

Fig. 6 shows that there are some sensible generic statements one can make about nonlinear wave packets described by the NLS equation. The first is that for the defocusing case, the nonlinearity really does accelerate the zonal spreading of the wave packet. This is shown even more dramatically in Fig. 7 which is the same except that the initial amplitude was increased by a factor of ten: almost all the widening of the envelope is due to nonlinearity.

However, it is important to remember always that the prediction of "fasterthan-linear" or "superlinear" dispersion is an asymptotic prediction, guaranteed only in the limit $t \rightarrow \infty$. A counterexample to the statement "a packet solution to the defocusing NLS equation always spreads" is provided by the "anti-soliton". This is defined to be a defocusing solution which at some time $t_{0}$ has the shape and amplitude of the NLS solitary wave for the focusing case, that is, for the NLS equation with the opposite sign in front of the nonlinear term. The anti-soliton is a useful special case because nonlinearity and dispersion are of equal strength at $t=t_{0}$ so that both effects are prominent. More 

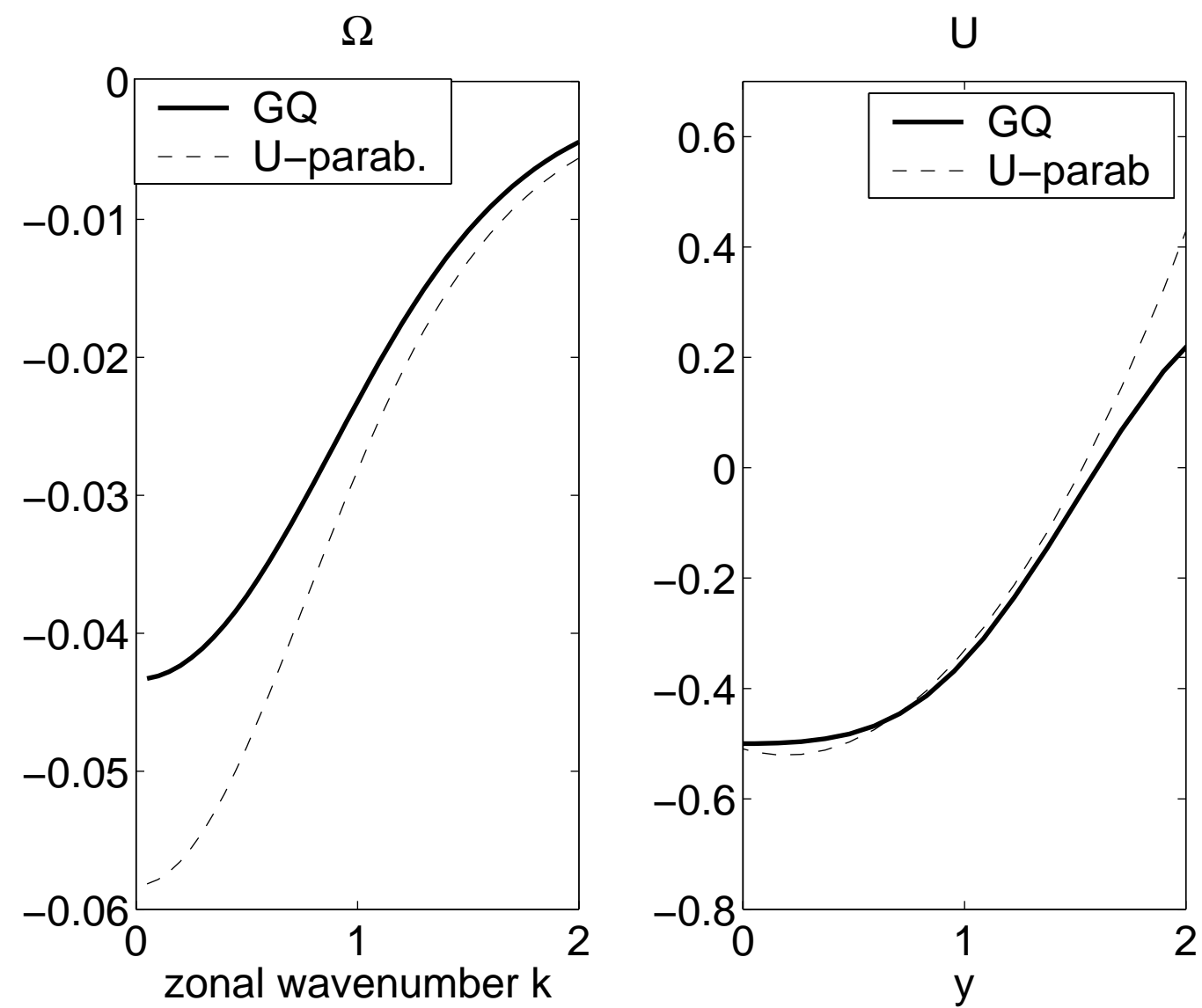

Figure 5: The right panel compares two similar but slightly different mean currents: the "Gaussian-Quartic" east-west flow (solid) and the "Unsymmetrical Parabola" (dashed). The left panel shows that the differences in $\Omega$ are greater than those of the mean currents themselves. More alarmingly, the parameter $\Omega$, which is a constant in second order perturbation theory in the shear strength, varies rather dramatically with wavenumber $k$. The dispersion is extremely weak for $k=2$. 


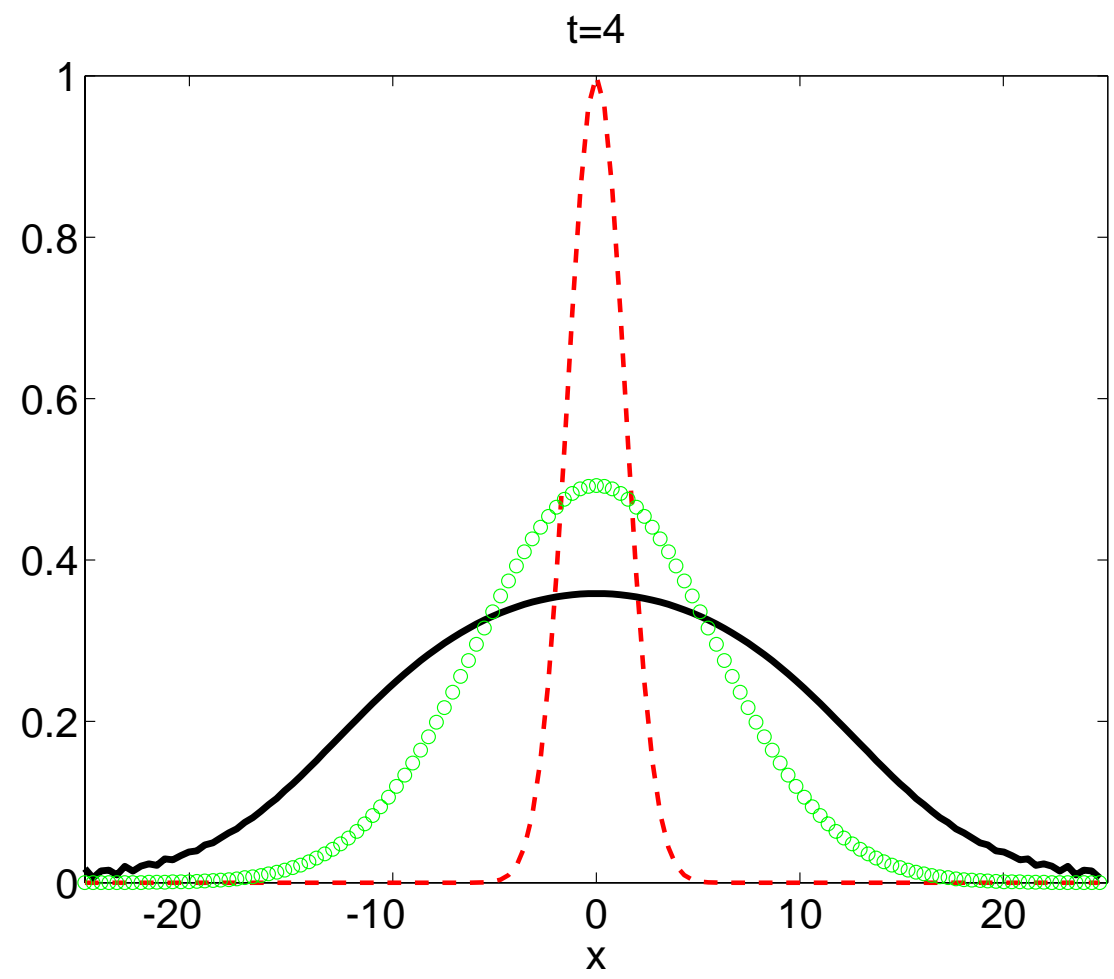

Figure 6: Solution to the NLS equation after rescaling to the canonical form $i w_{t}+w_{x x}-2|w|^{2} w=0$ for the intial condition $w(x, 0)=\exp \left(-x^{2} / 4\right)$, which is the dashed curve. Solid: the nonlinear solution at $t=4$. Circles: solution to the linear NLS equation $i w_{t}+w_{x x}=0$.

important, the anti-soliton is symmetric in time with respect to $t=t_{0}$, that is, $A\left(x, t-t_{0}\right)=A\left(x, 2 t_{0}-t\right)$ for all $t$ as illustrated in Fig. 8.

It follows that if $t_{0}>0$, the anti-soliton will spontaneously self-focus for $t \in\left[0, t_{0}\right]$ and will only widen for larger times. 


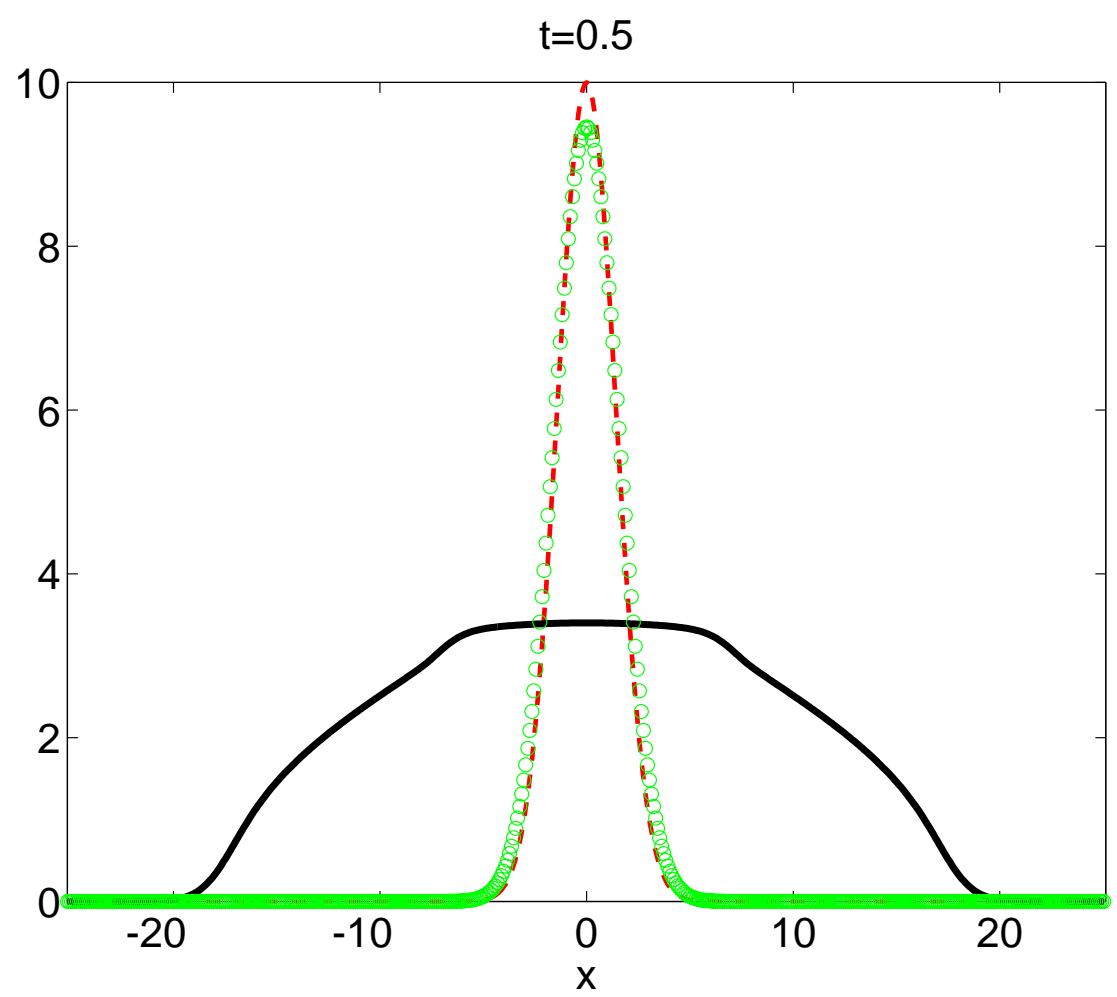

Figure 7: Same as previous figure except that the initial amplitude was increased by a factor of ten, and the final time was reduced to $t=1 / 2$. The linear solution is almost indistinguishable from the initial condition, but the nonlinear solution has dispersed rapidly. 


\section{Anti-soliton: $|u(x, t)|$}

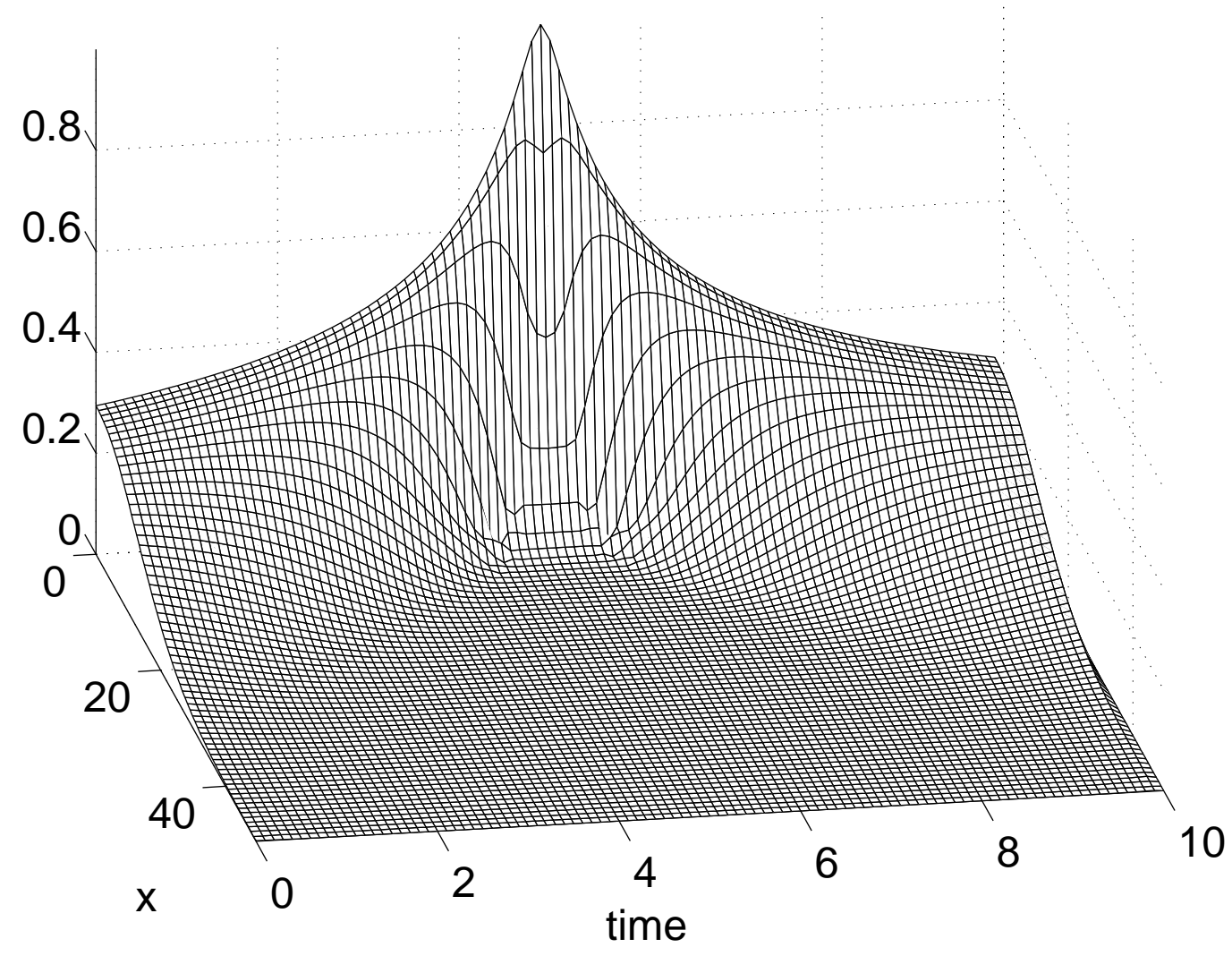

Figure 8: Absolute value of anti-soliton. At $t=t_{0}, u\left(x, t ; t_{0}\right)=\operatorname{sech}(x)$. The particular case $t_{0}=5$ is illustrated, but the shape of the antisoliton is the same for all $t_{0}$ because $u\left(x, t ; t_{0}\right)=u\left(x, t-t_{0} ; t_{0}=0\right)$ for all $\left(x, t, t_{0}\right)$. Only positive $x$ is shown because the anti-soliton is symmetric with respect to $x=0$, that is, $u\left(x, t ; t_{0}\right)=u\left(-x, t ; t_{0}\right)$ for all $\left(x, t, t_{0}\right)$. 


\section{Analysis}

Let $W$ denote the width of the envelope and let $U$ denote the magnitude of $|A(x, t)|$, expressed in terms of the usual nondimensional length and time scales for equatorial oceangraphy defined by Eq. (5). Scale analysis shows the time scale for dispersion is

$$
\mathcal{T}_{\text {linear dispersion }} \sim \frac{W^{2}}{3 \Omega \delta^{2} k}
$$

where $\delta^{2} \Omega$ is the strength of the dispersive, second order correction to the phase speed and $k$ is the central packet wavenumber. The multiple scales expansion is accurate only if $W k>>1$, at least formally. The ratio of the nonlinear term in the Schrödinger equation to the linear dispersive term is

$$
\mathcal{N} \sim \frac{U^{2} W^{2}}{48 \Omega^{2} \delta^{4} k^{2}}
$$

Note that $\delta$ is a parameter used only to scale the perturbation theory; we can choose $\Omega$ to be $\mathrm{O}(1)$ and $\delta$ small or alternatively set $\delta=1$ and then $\Omega$ will be small, proportional to the square of the mean shear. Only the product $\delta^{2} \Omega$ appears in the physics. In the next section, we take the simplest approach by setting $\delta=1$ and allowing $\Omega$ to be small.

The Kelvin wave in the first baroclinic mode will cross the width of the Pacific in about forty nondimensional time units. It follows that linear dispersion, due to the second derivative term in the Schroedinger equation, will significantly widen and reshape the wave envelope only if the packet is excited not too close to the coast and if the dispersive time scale is $\mathrm{O}(10)$ or smaller. If the nonlinear/linear ratio $\mathcal{N}$ is small compared to one, then the packet evolution is LINEAR. If, however, $\mathcal{N} \sim O(1)$, then both linear and nonlinear dispersion are important.

No simple analytical solutions for the defocusing case are known. In the next section, we shall use scale analysis and numerical modelling to look at particular cases.

The timescale for linear dispersion is then

$$
\mathcal{T}_{\text {linear dispersion }} \approx 7 \frac{W^{2}}{k}
$$

It follows that linear dispersion will significantly reshape the wave envelope in the month or two required for the packet to reach the east coast of the Pacific Ocean only if

$$
\frac{W^{2}}{k} \leq 1 / 3 \quad \leftrightarrow \quad W^{2} \leq k / 3
$$

To be accurate, the multiple scales perturbation theory requires that the envelope should be wide compared to the length scale of the carrier wave. This imposes the further requirement that

$$
k W>>1
$$


Fig. 9 is a map of the two-dimensional $(k, W)$ parameter space. The shaded region shows where the perturbation theory is credible and at the same time, the wavepacket widens significantly (say by a factor of two) in the trans-Pacific crossing time.

The implication is that it is equatorial waves with rather high carrier wavenumbers of say, $k=2$ or larger, where our theory is applicable. This corresponds to a nondimensional carrier wavelength of $\pi$ or roughly one thousand kilometers in zonal wavelength.

Unfortunately, this is rather a severe strain on current observing systems. The TOPEX/Poseidon data set of Susanto et al. has a resolution of 1 degree in latitude (about $100 \mathrm{~km}$ ) and 2.8 degrees in longitude (roughly $300 \mathrm{~km}$ ). Thus, the interesting wave packets have a density of only about three data points per wavelength in the TOPEX/Poseidon data! We cannot expect such data to accurately characterize wavelengths with carrier waves of $k \approx 2$ or shorter.

A second difficulty in applying our theory to the real world is that the ratio of the nonlinear to the linear term in the $N L S$ equation scales as the fourth power of the perturbation parameter. Thus, when the perturbation parameter is halved, the ratio of nonlinear term to dispersive term in the NLS equation, $\mathcal{N}$ defined by Eq. (55) above, will change by a factor of sixteen! Fortunately, t he situation is not as bad as it seems because the dispersive term is inversely proportional to the square of the zonal scale of the envelope and the nonlinear term is proportional to the square of the envelope, relative to the other terms in the equation. Thus, the relative magnitude of the terms can be restored by halving the spatial scale of the initial envelope while simultaneously doubling its amplitude. Still, it is clear that modest errors in estimating either the initial envelope or the mean shear flow will dramatically alter the answer.

\section{Numerical Solutions of the Shallow Water Equa- tions}

Despite the absence of good observations of equatorial wave packets, we performed some numerical simulations of weakly nonlinear Kelvin wave packets. Our model is that of Boyd(1998b), which employs periodic boundary conditions in $x$ (longitude) and eighth-order centered spatial differences with third-order Adams-Bashforth time-stepping. No damping is used.

We integrated from the same initial conditions, first without a mean flow and then a second time with the Gaussian-Quartic mean current defined by Eq. 50. The initial condition is [excluding the mean current and height, if any]

$$
u(x, y, t)=\phi(x, y, t)=\frac{1}{20} \exp \left(-\frac{1}{2} y^{2}\right) \cos (x) \exp \left(-\frac{1}{25} x^{2}\right)
$$

Figs. 10 and 12 show that in the absence of a mean flow, the tallest crest of the wavepacket rapidly breaks, and the shorter crests are steepening on the eastward sides, and will break, too, in time. In contrast, the same wave packet 


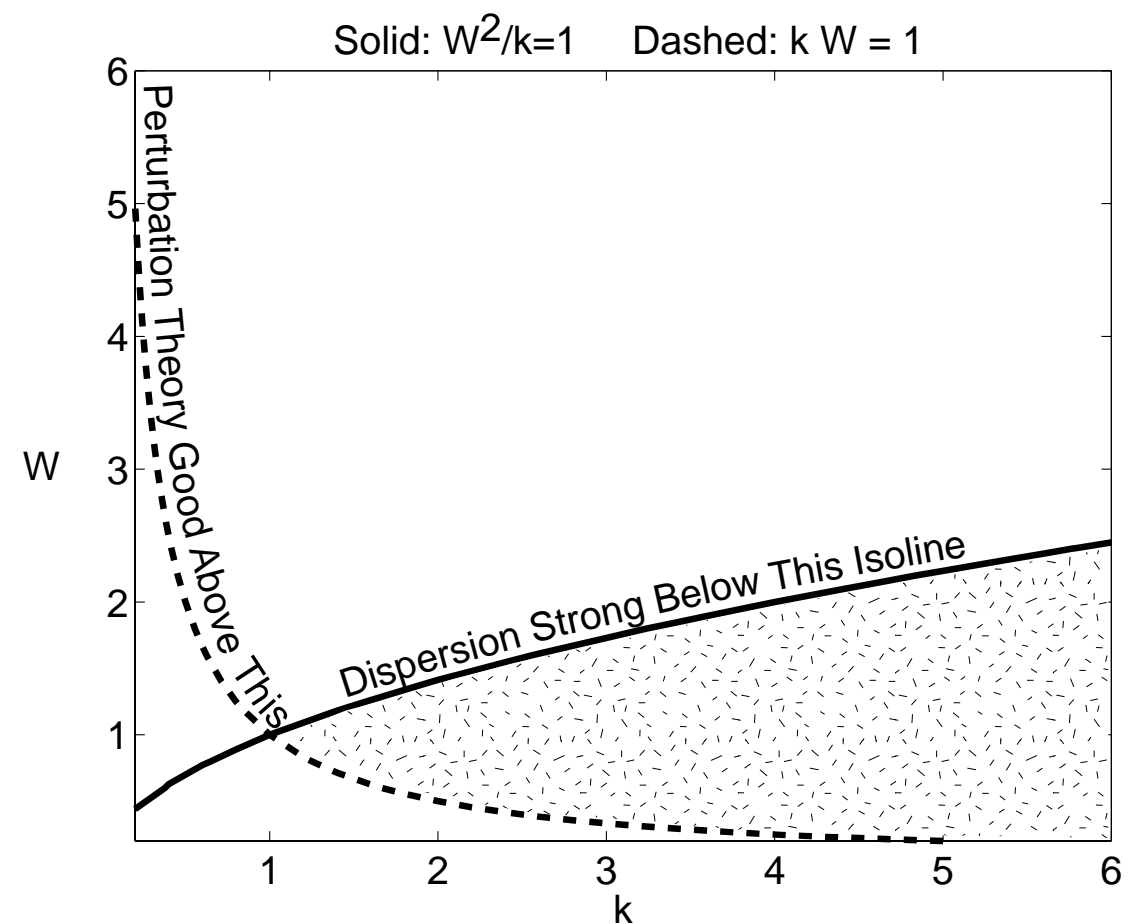

Figure 9: The $k-W$ plane in parameter space where $W$ is the width of the envelope and $k$ is the wavenumber of the carrier wave. The solid contour is the curve $W^{2} k=1$. Below this curve, the packet will significantly disperse on the time scale required for a Kelvin wave to cross the Pacific Ocean. (Wide envelopes, that is, large $W$, will widen further only on a very long time scale.) The dashed contour denotes where $k W=1$. Above and to the right of this curve, the multiple scales/weakly nonlinear perturbation theory is accurate. (Strictly speaking, the perturbation theory is the asymptotic limit $k W \rightarrow \infty$, which is movement towards the upper right corner of the diagram, but in practice such theories are at least qualitatively accurate even when the perturbation parameter $1 /(k W)$ is not particularly small.) The shaded region shows the interesting region where both the perturbation theory is believable and the wavepacket is dispersing. 
in the presence of our standard Gaussian-Quartic mean flow shows no visible signs of breaking.

Fig. 12 shows the initial conditions, too, to illustrate that at $t=16$, the wave packet has dispersed only a little, with or without a mean current. The interesting effect of nonlinearity is not faster-than-linear dispersion, but rather the absence of breaking.

Experiments at larger amplitudes invariably gave wave-breaking. Were the $\mathrm{KdV}$ dynamics applicable for all wavenumbers and shear strengths (as we have already seen is not true), then it would always be possible to forestall breaking by making the crests sufficiently narrow. In other words, in KdV dynamics, breaking can always be suppressed by increasing the carrier wavenumber $k$. The same apparently is not true for Kelvin waves.

To quantify this, we ran a series of experiments which began with

$$
u(x, t)=\mathcal{A} \sin (k x)
$$

for various $\mathcal{A}$ and $k$ where $\mathcal{A}$ and $k$ are constants. We found that breaking always occurred for sufficiently large amplitude $\mathcal{A}$ for all $k$. Fig. 13 shows the boundary. Wavepackets with non-constant envelopes also break for sufficiently large amplitude, but we have not attempted to map the whole parameter space.

At present, there is no theory for this "dispersion crisis" in which even very narrow wavepackets (or waves) cannot resist sufficiently strong nonlinearity. This phenomenon is yet another sign that our $\mathrm{KdV} /$ shear-strength-expansion is restricted to rather small amplitude and weak mean flow.

\section{Summary}

Our analysis of nonlinear Kelvin packets yields a mixed bag of results. Some assertions can be made with confidence:

1. The nonlinear dynamics is described, for weak nonlinearity and wide envelopes, by the envelope evolving as a solution to the Nonlinear Schroedinger (NLS) equation.

2. To second order in the strength of the mean shear flow, the NLS equation is

$$
i\left(A_{01}\right)_{\tau}+3 \Omega \delta^{2} k\left(A_{01}\right)_{\zeta \zeta}-\frac{1}{16 \Omega \delta^{2} k}\left|A_{01}\right|^{2} A_{01}=0
$$

where $A_{01}$ is the envelope, $\zeta$ is a coordinate that travels eastward at the linear group velocity, $k$ is the carrier wavenumber and $\Omega$ is given by Eq. 27 (Sec. 3). ( $\Omega$ is independent of $k$ in the perturbative approximation.)

3. The near-resonance of Kelvin waves of all wavenumbers simplifies the derivation since only the self-interaction of the Kelvin wave is important for weak shear. In particular, the NLS equation can be derived from the Korteweg-deVries equation (Boyd, 1984) that describes weakly nonlinear Kelvin waves of arbitrary shape, not just wavepackets. 

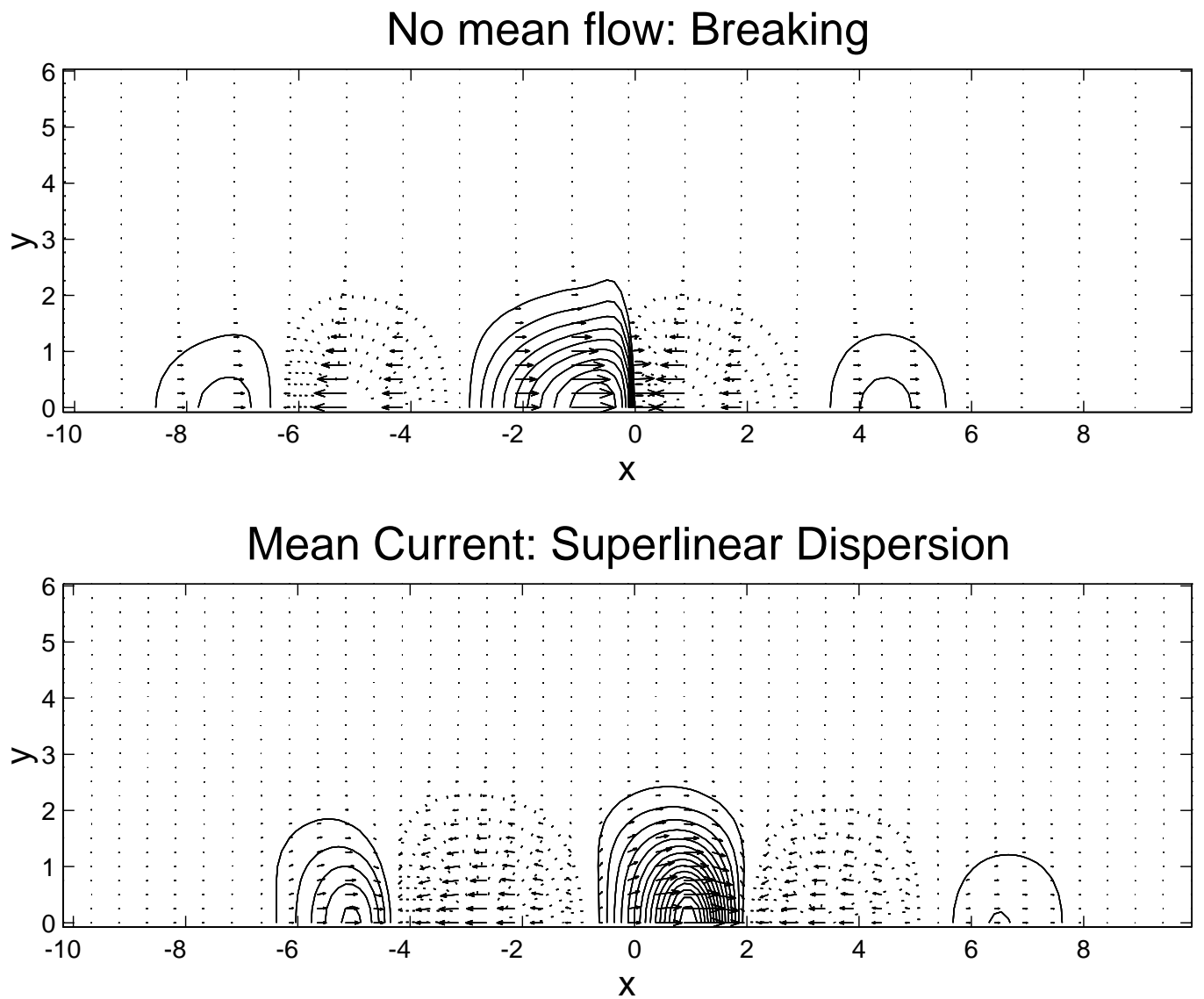

Figure 10: Contour plots of the height anomaly (with the zonal mean subtracted) with a contour interval of 0.005 , superimposed upon a quiver plot of the horizontal velocities. Flows are shown at $t=16$, which is three to four weeks. Top: no mean flow; the packet steepens and then breaks. The very concentrated contours at $x=0$ are the signature of steep east-west gradients (the frontal zone). Bottom: same except the mean flow is the Gaussian-Quartic combination of jets. Computed on the domain $x \in[-16 \pi, 16 \pi] \otimes y \in[0,2 \pi]$. The time step was $1 / 100$, the spatial grid was $800 \otimes 25$. 

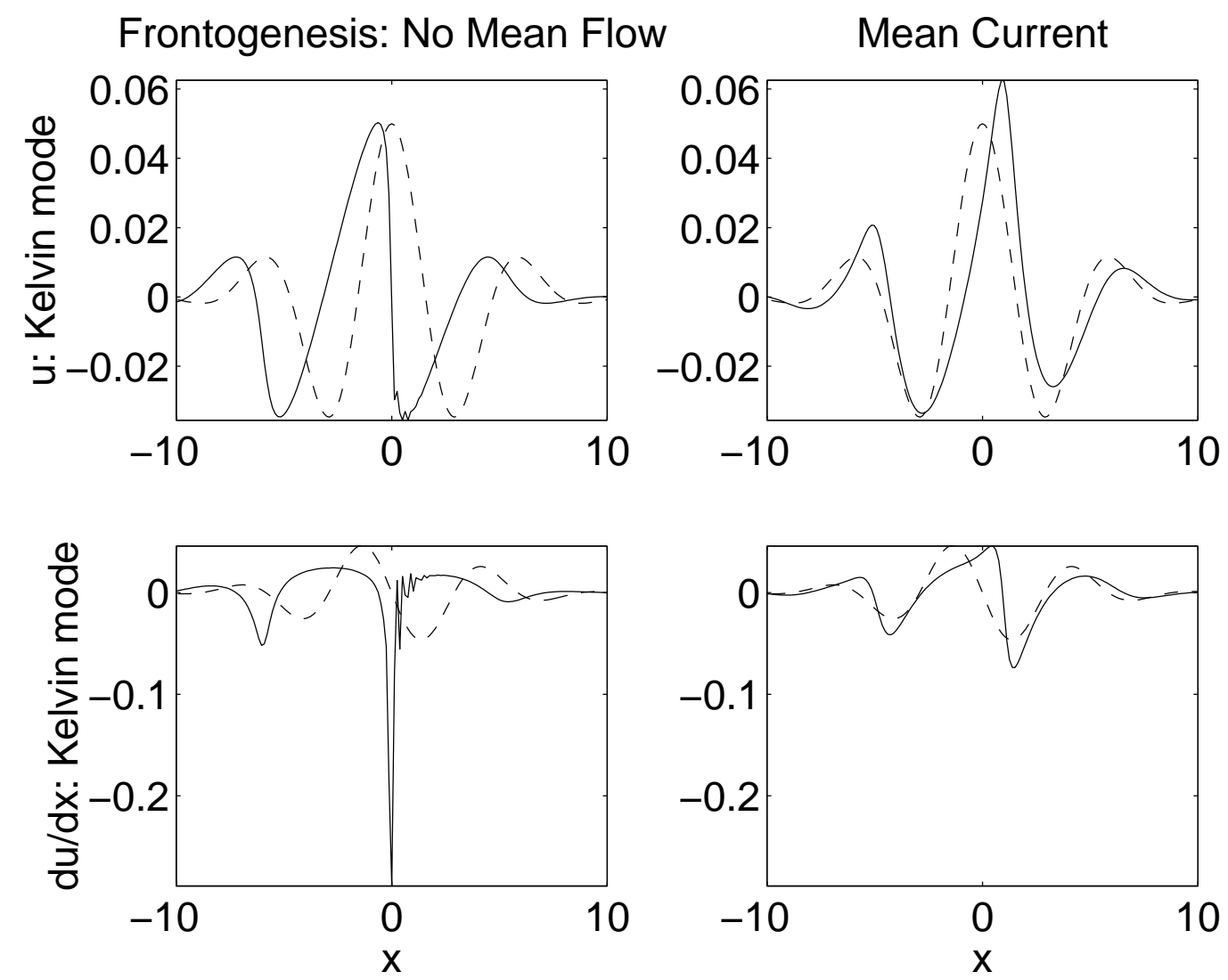

Figure 11: Same as previous figure except that only the projection of the eastwest velocity $u$ onto the Kelvin mode is shown. The solution at $t=16$ (about a month) is solid; the initial condition is dashed. The left panels show the Kelvin wave amplitude (top) and its east-west derivative (bottom) when there is no shear. The derivative (left bottom) shows a huge spike where the central peak has developed a vertical slope. With the Gaussian-quartic mean flow (right panels), there is no breaking and the longitudinal slope evolves but does not grow. 


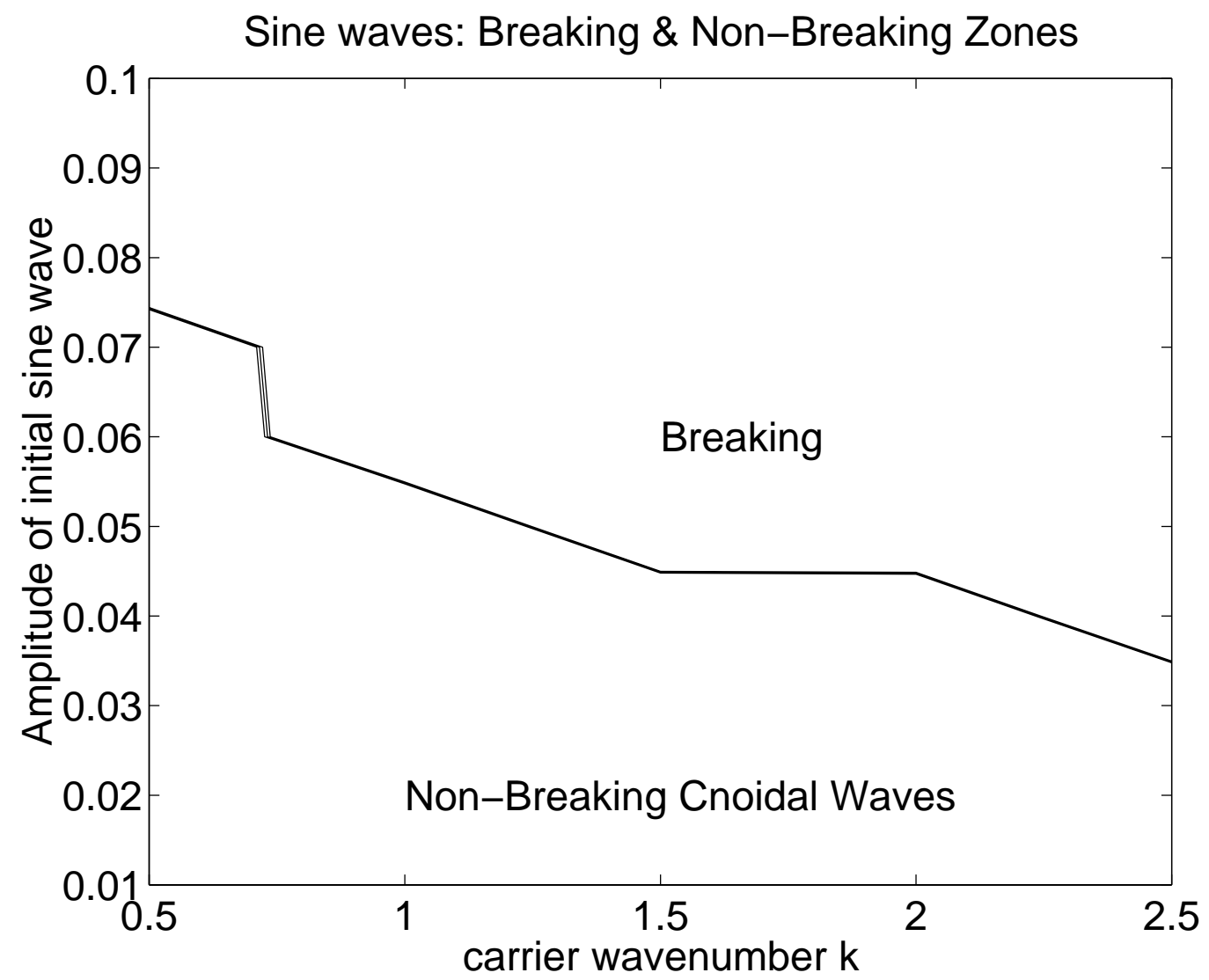

Figure 12: The solid line is the boundary between breaking and nonbreaking Kelvin wave packets when the initial condition is a Kelvin wave which is a sine wave, $\mathcal{A} \sin (k x)$, in longitude. The initial disturbance's wavenumber $k$ is the horizontal axis and its amplitude $\mathcal{A}$ is the vertical axis. 
4. The sign of the NLS equation is such that it is always defocusing for Kelvin waves, implying that the envelope spreads faster than in a linear calculation.

There are some difficulties in applying the theory presented here to the ocean:

1. Data are as yet inadequate to quantify the envelope width and carrier wavenumber, save perhaps in order of magnitude

2. The ratio of the nonlinear coefficient to the dispersive coefficient in the NLS equation varies as the fourth power of the strength of the shear, implying great sensitivity to the mean current.

3. Numerical solutions of the linear eigenvalue problem for waves on a mean current show that the dispersion parameter $\Omega$, which is independent of carrier wavenumber $k$ in the perturbation theory, in fact varies strongly with wavenumber. This suggests the expansion in powers of the shear strength $\delta$ is accurate only for weaker currents than those actually observed.

4. Numerical solutions of the full shallow water equations show that large and moderate amplitude packets have breaking crests, even though the $\mathrm{KdV} / \mathrm{NLS}$ theory states that breaking can always be suppressed by making the carrier wavenumber sufficiently large. This is contrary to the $\mathrm{KdV} /$ shear-strength-expansion theory.

5. Transient focusing is possible in the NLS equation even when the asymptoticin-time behavior is guaranteed to be faster-than-linear dispersion.

Open problems:

1. Why does dispersion fail to prevent breaking when the initial wave packet or bell soliton shape is larger than some threshold?

2. This is a subset of a larger question, which is how to describe Kelvin wave packets when the mean currents and/or the packet amplitude are too large for the analytic theories derived here to be accurate.

The NLS theory can be extended by replacing the shear-perturbation theory by numerical computation of a sequence of linear boundary value problems. We have not done this here because (i) it is complicated and (ii) the NLS equation cannot explain the frontogenesis and breaking that inevitably occur at large amplitude. What is really needed is a larger-amplitude extension of the NLS equation with additional terms.

Thus, we have developed a consistent theory for Kelvin wavepackets for weak currents and small amplitude. Improvements in observations will someday allow insertion of the correct numbers into the theory. However, theoretical improvements and more numerical experiments are needed to understand what really happens for moderate amplitude and the strong mean flows of the tropical ocean. 
Equatorial wave disturbances occur on other planets, too. Transient Kelvin waves were observed, for example, in the numerical modelling of Jovian equatorial hot spots and plumes by Showman and Dowling(2000). Perhaps the weak shear/small amplitude theory will be more accurate on Jupiter or Mars.

\section{Acknowledgments}

This work was supported by the National Science Foundation through grant OCE9521133. Dr. Chen is grateful for a three-year graduate fellowship provided by the Department of Education, Taiwan, R. O. C. We also appreciate the reviews by Dennis Moore and an anonymous referee.

\section{References}

Ablowitz, M. J. and H. Segur, 1981: Solitons and the Inverse Scattering Transform. Philadelphia, Pennsylvania: SIAM.

Anderson, D. L. T., 1973: An ocean model using parabolic cylinder functions. Geneva: World Meteorological Organization.

Boyd, J. P., 1978a: The effects of latitudinal shear on equatorial waves, Part I: Theory and methods Journal of the Atmospheric Sciences, 35, 2236-2258.

Boyd, J. P., 1978b: The effects of latitudinal shear on equatorial waves, Part II: Applications to the atmosphere Journal of the Atmospheric Sciences, 35, 2259-2267.

Boyd, J. P., 1980: The nonlinear equatorial Kelvin wave Journal of Physical Oceanography, 10, 1-11.

Boyd, J. P., 1983a: Equatorial solitary waves, Part II: Envelope solitons Journal of Physical Oceanography, 13, 428-449.

Boyd, J. P., 1983b: Second harmonic resonance for equatorial waves Journal of Physical Oceanography, 13, 459-466.

Boyd, J. P., 1984: Equatorial solitary waves, Part IV: Kelvin solitons in a shear flow Dynamics of Atmospheres and Oceans, 8, 173-184.

Boyd, J. P., 1998a: Weakly Nonlocal Solitary Waves and Beyond-All-Orders Asymptotics: Generalized Solitons and Hyperasymptotic Perturbation Theory, volume 442 of Mathematics and Its Applications. Amsterdam: Kluwer $608 \mathrm{pp}$.

Boyd, J. P., 1998b: High order models for the nonlinear shallow water wave equations on the equatorial beta-plane with application to Kelvin wave frontogenesis Dynamics of Atmospheres and Oceans, 28(2), 69-91. 
Boyd, J. P., 1999: The Devil's Invention: Asymptotics, superasymptotics and hyperasymptotics Acta Applicandae, 56(1), 1-98.

Brown, E. D., S. Buchsbaum, R. E. Hall, J. P. Penhune, K. F. Schmitt, K. M. Watson, and D. C. Wyatt, 1989: Observations of a nonlinear solitary wavepacket in the Kelvin wave of a ship J. Fluid Mech., 204, 263-293.

Chen, G.-Y., 1998: Application of Weak Nonlinearity in Ocean Waves $\mathrm{PhD}$ dissertation, University of Michigan, Department of Atmospheric, Oceanic and Space Sciences.

Delcroix, T., J. Picaut, and G. Eldin, 1991: Equatorial Kelvin and Rossby waves evidenced in the Pacific Ocean through Geosat sea level and surface current anomalies J. Geophys. Res., 96(C10), 3249-3262.

Desurvire, E., 1994: The Golden Age of optical fiber amplifiers Physics Today, 47, 20-27.

Fedorov, A. V. and W. K. Melville, 2000: Kelvin fronts on the equatorial thermocline J. Phys. Oceangr., 30 in press.

Glass, A. M., 1993: Fiber optics Physics Today, 46, 34-38.

Hasegawa, A. and F. Tappert, 1973: Applied Physics Letters, 23, 142.

Kessler, W. S., M. J. McPhaden, and K. M. Weickmann, 1995: Forcing of intraseasonal kelvin waves in the equatorial pacific J. Geophys. Res., 100(C6), 10613-10631.

Lake, B. M., H. C. Yuen, H. Rungaldier, and W. E. Ferguson, 1977: Nonlinear deep-water waves: theory and experiment. Part 2. Evolution of a continuous wave train Journal of Fluid Mechanics, 83, 49-74.

Long, B. and P. Chang, 1990: Propagation of an equatorial Kelvin wave in a varying thermocline Journal of Physical Oceanography, 20, 1826-1841 Classical solitons and breaking waves.

Marshall, H. G. and J. P. Boyd, 1987: Solitons in a continuously stratified equatorial ocean Journal of Physical Oceanography, 17, 1016-1031.

Mollenauer, L. F., R. H. Stolen, and J. P. Gordon, 1980: Experimental observation of picosecond pulse narrowing and solitons in optical fibers Physical Review Letters, 45(13), 1095-1099.

Moore, D. W. and S. G. H. Philander, 1977: Modelling of the tropical oceanic circulation In E. D. Goldberg (Ed.), The Sea, number 6 (pp. 319-361). New York: Wiley.

Ripa, P., 1982: Nonlinear wave-wave interactions in a one-layer reduced-gravity model on the equatorial beta-plane Journal of Physical Oceanography, 12, $97-111$. 
Ripa, P., 1985: Nonlinear effects in the propagation of Kelvin pulses across the Pacific Ocean In L. Debnath (Ed.), Advances in Nonlinear Waves (pp. 43-56). New York: Pitman.

Showman, A. P. and T. E. Dowling, 2000: Nonlinear simulations of Jupiter's $5-\mu m$ hot spots Science In press.

Susanto, R. D., Q. N. Zheng, and X. H. Yan, 1998: Complex singular value decomposition analysis of equatorial waves in the Pacific observed by TOPEX/Poseidon altimeter J. Atmos. Ocean Technology, 15(3), 764-774.

Tribbia, J. J., 1984: A simple scheme for high-order nonlinear normal initialization Monthly Weather Review, 112, 278-284.

Whitham, G. B., 1974: Linear and Nonlinear Waves. New York: John Wiley \& Sons.

Yuen, H. C. and B. M. Lake, 1975: Nonlinear deep water waves: theory and experiment Physics of Fluids, 18, 956-960.

Zheng, Q., X. Yan, C. Ho, and C. Tai, 1995: Observation of equatorially trapped waves in the Pacific using GEOSAT altimeter data Deep Sea Research, 42(5), 797-817. 